\title{
Experimental analysis of robot-assisted needle insertion into porcine liver
}

\author{
Wendong Wang ${ }^{\mathrm{a}, \mathrm{b},{ }^{*}}$,Yikai Shi ${ }^{\mathrm{a}}$, Andrew A. Goldenberg ${ }^{\mathrm{b}}$, Xiaoqing Yuan ${ }^{\mathrm{a}}$, Peng Zhang ${ }^{\mathrm{a}}$, \\ Lijing $\mathrm{He}^{\mathrm{a}}$ and Yingjie Zou ${ }^{\mathrm{a}}$ \\ ${ }^{a}$ School of Mechanical Engineering, Northwestern Polytechnical University, Xi'an, Shaanxi, P.R. \\ China \\ ${ }^{b}$ Department of Mechanical \& Industrial Engineering, University of Toronto, Toronto, ON, Canada
}

\begin{abstract}
How to improve placement accuracy of needle insertion into liver tissue is of paramount interest to physicians. A robot-assisted system was developed to experimentally demonstrate its advantages in needle insertion surgeries. Experiments of needle insertion into porcine liver tissue were performed with conic tip needle (diameter $8 \mathrm{~mm}$ ) and bevel tip needle (diameter $1.5 \mathrm{~mm}$ ) in this study. Manual operation was designed to compare the performance of the presented robot-assisted system. The real-time force curves show outstanding advantages of robot-assisted operation in improving the controllability and stability of needle insertion process by comparing manual operation. The statistics of maximum force and average force further demonstrates robot-assisted operation causes less oscillation. The difference of liver deformation created by manual operation and robot-assisted operation is very low, $1 \mathrm{~mm}$ for average deformation and $2 \mathrm{~mm}$ for maximum deformation. To conclude, the presented robot-assisted system can improve placement accuracy of needle by stably control insertion process.
\end{abstract}

Keywords: Robot-assisted system, needle insertion, manual operation, insertion force

\section{Introduction}

Liver is the most metabolically complex organ and performs an estimated 500 functions [1]. Currently, liver disease across the world is wide-spread because of parasitic and other diseases [2,3]. Meanwhile, it is the most frequently injured abdominal organ due to injuries generally caused by motor vehicle accidents and falls from heights, which result in rapid decelerations [4, 5]. Therefore, many modern clinical procedures require insertion of needles into soft tissues. One of the main challenges related to needle insertion is the accurate placement of the needle within the lesion because the liver is subject to respiratory motion [6]. Therefore, needle insertion into liver requires advanced skills to make a plan for insertion position and angle taking into account of organ deformation [7].

Mechanical characterization of liver tissue can be highly beneficial in the management of liver diseases and injuries $[8,9]$. The experimental data of in vivo mechanical behavior of human liver allow a quantitative analysis of the relationship between mechanical response and modifications of tissues micro-structure [10]. Clinical research has shown that blunt liver injury is associated with

\footnotetext{
* Address for Correspondence: Wendong Wang, School of Mechanical Engineering, Northwestern Polytechnical University, 127 West Youyi Road, Xi'an, Shaanxi. 710072. P.R. China. Tel.: +86 029-88494893; Fax: +86 029-88494893; E-mail: wdwang@nwpu.edu.cn.
} 


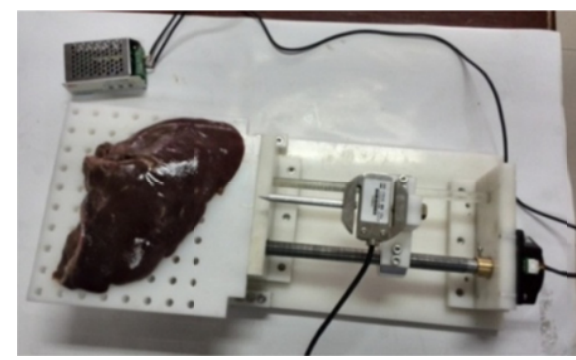

(a)

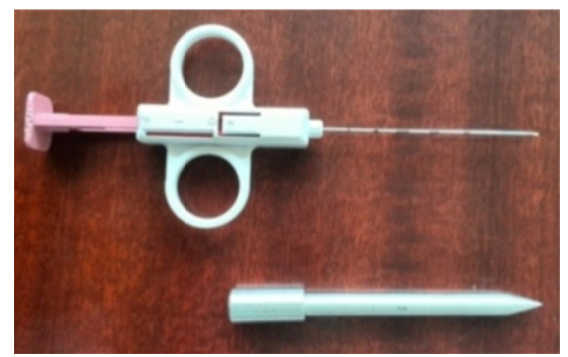

(b)

Fig. 1. Prototype of the robot-assisted needle insertion system with porcine liver (a); (top) bevel tip needle and (bottom) conic tip needle (b).

mortality rates as high as $30 \%$ in cases requiring operative intervention [11].

Recently, research on robotic systems also has been conducted with the purpose of improving needle placement accuracy and expansion of approach path. Maier-Hein, et al [12] introduced a needle-based navigation concept for CT-guided punctures of the liver and aimed to investigate whether the overall insertion error could be further reduced by replacing the user with a medical robot for the needle orientation and insertion. An intelligent in-bore-operated robot was developed for the purpose of transperineal prostate interventions concerning intelligent control of the needle [13]. All the above research work focused on the navigation of needle insertion to improve placement accuracy. To the best of author's knowledge, there is no existing work discussing how the robot-assisted system improves needle insertion process. The goal of our study is to experimentally demonstrate the advantages of robot-assisted needle insertion compared with manual operation.

\section{Methodology}

\subsection{Experimental setup}

The robot-assisted needle insertion system (see Figure 1(a)) was developed to mount porcine liver sample and drive insertion needles. A force sensor (CFBLSM, Gaoling sensing system Co., Ltd.) was chosen to build sensing system and collect experiment data. An ultrasonic motor (TRUM-60, Chunsheng Co., Ltd) was selected as the actuator of the insertion system. The mounting plates of the insertion system were designed to be adjustable in vertical and horizontal directions to fix different samples. Meanwhile, this design enables the needle reach to any point of the liver sample. To avoid the influence on insertion force and deformation from insertion speed, a constant speed of $70 \mathrm{~mm} / \mathrm{min}$ was employed in all robot-assisted operations. To compare the difference of insertion process caused by different needles, a bevel tip needle (SuperCore ${ }^{\mathrm{TM}}$ instrument, $1.5 \mathrm{~mm}$ in diameter) and a conic tip needle ( $8 \mathrm{~mm}$ in diameter, custom-made) were prepared as shown in Figure 1(b) to puncture the freshly harvested porcine livers with the presented system and hands respectively.

\subsection{Preparation of the samples}

A whole porcine liver sample was taken immediately post sacrifice. The liver was divided into two parts due to the space limitation of mounting plate and tested as a half to minimize the blood loss and possible changes in the boundary conditions. Markers of $5 \mathrm{~mm}$ in diameter were placed on the surface of the porcine liver during insertion so that experiments can be repeated for different locations in one 


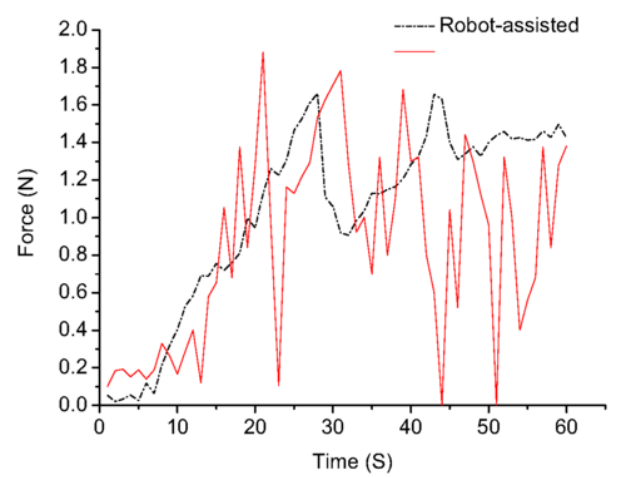

Fig. 2. Insertion force curves with bevel tip needle.

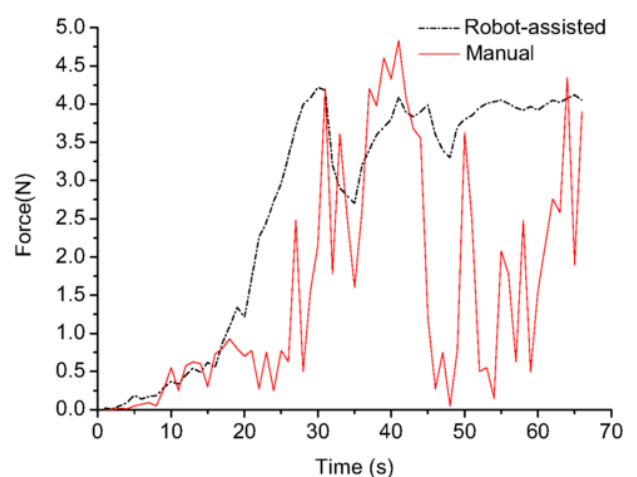

Fig. 3. Insertion force curves with conic tip needle.

sample. All tests were performed at room temperature, and the statistic data were taken based on ten tests with the same needle insertion operations. The sample was immersed in physiological saline to prevent drying before the experiment.

\section{Results}

\subsection{Comparison of insertion process}

The liver insertion processes of manual and robot-assisted operation were compared by recording real-time force. Insertion force-time curves created with bevel tip needle and conic tip needle were given in Figures 2 and 3. With bevel tip needle, the maximum insertion force is $1.88 \mathrm{~N}$ for manual operation and $1.65 \mathrm{~N}$ for robot-assisted operation, it is $4.85 \mathrm{~N}$ and $4.22 \mathrm{~N}$ respectively with the conic tip needle. From the above force values, the robot-assisted operation did not affect the insertion process from the point of view of maximum force and average force, instead, it significantly smoothed the real-time force curves obtained with both bevel tip needle and conic tip needle. Moreover, once the insertion started, the insertion force rapidly increased before the liver was punctured through in manual and robot-assisted operation. The statistics shown in Figure 4 indicates the difference between maximum force and average force with conic tip needle is $1.1 \mathrm{~N}$ for robot-assisted operation and $1.9 \mathrm{~N}$ for manual operation, which further demonstrates robot-assisted operation causes less oscillation.

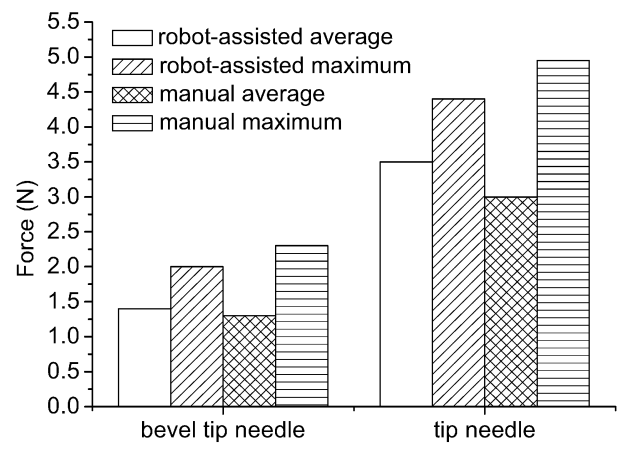

Fig. 4. Statistics of liver insertion force.

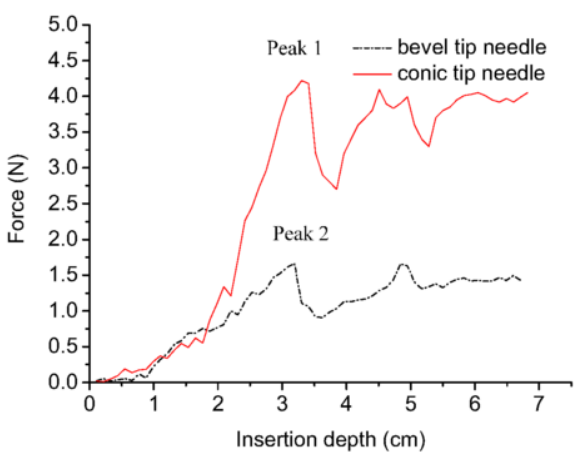

Fig. 5. Insertion force vs. depth under robot-assisted operation. 


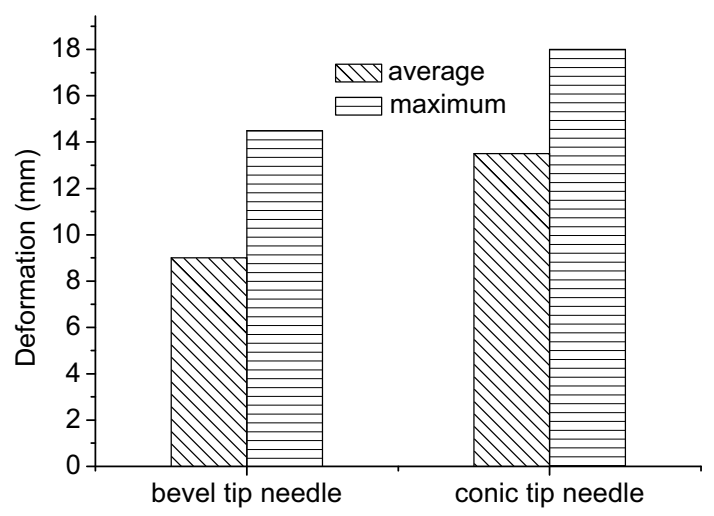

(a) manual operation

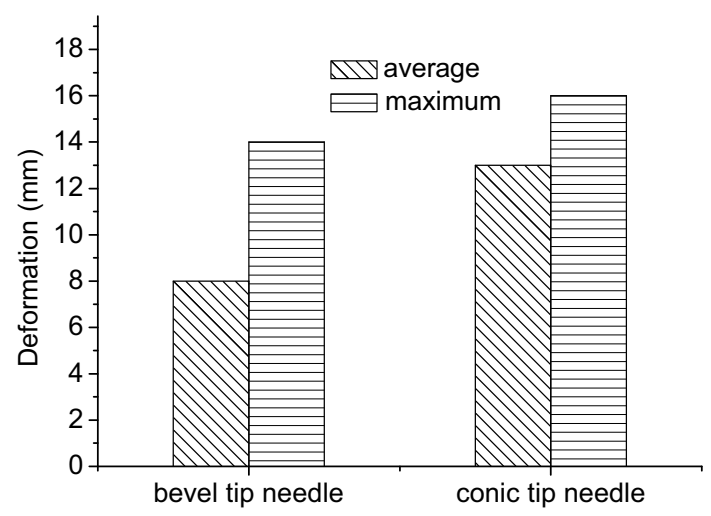

(b) robot-assisted operation

Fig. 6. Statistics of liver deformation.

The insertion depth of robot-assisted operation was calculated to investigate the relation between insertion force and depth. In our study, the insertion operations were implemented with a depth of $6.5 \pm 0.5 \mathrm{~cm}$. In Figure 5, the force versus deformation reveals one primary puncture and subsequent internal punctures. Before the peak (Peak 1 and Peak 2) point, the main puncture event is designated by a peak in force after a steady rise, followed by a sharp decrease.

\subsection{Analysis of liver deformation}

The deformation of liver sample during needle insertion was investigated in this study. Statistics of liver deformation in manual operation (see Figure 6(a)) shows the maximum deformation is $18 \mathrm{~mm}$ for conic tip needle and $14.5 \mathrm{~mm}$ for bevel tip needle, and the average deformation is $13 \mathrm{~mm}$ for conic tip needle and $9 \mathrm{~mm}$ for bevel tip needle. The deformation created by conic tip needle is around $45 \%$ larger than bevel tip needle because of the larger diameter. Comparing Figure 6(a) with Figure 6(b), the difference of liver deformation created by manual operation and robot-assisted operation is very low, $1 \mathrm{~mm}$ for average deformation and $2 \mathrm{~mm}$ for maximum deformation.

\section{Discussion}

The force data collected is a summation of stiffness, friction, and cutting forces. The stiffness force occurs before puncture of the capsule, and the friction and cutting forces occur after this main puncture. Cutting forces include the plastic deformation from the act of cutting as well as the force resulting from tissue stiffness at the tip of the needle. Likewise, friction force is also a function of the internal stiffness of the tissue [14]. From Figures 2 and 3, the steady rise of insertion force is mainly caused by stiffness, after the point Peak 1 and peak 2 appear, the insertion force mainly come from friction and cutting. Meanwhile, the insertion force varies with the needle diameter, and the bigger diameter causes the increase of insertion force. As the needle diameter increases, the more liver tissue is displaced and compressed in the vicinity of the needle. This increases the forces normal to the surface of the needle, leading to higher friction forces.

Comparing the force curves shown in Figures 2 and 3, the insertion process of robot-assisted operation is much more stable than manual operation as the force oscillation from robot-assisted operation is much smaller than manual operation. The force oscillation may cause deformation of soft 
tissue and displacement of insertion site. Therefore, it can explain the reason that robot-assisted system significantly improves the controllability and stability of needle during insertion into liver. Moreover, combining image guide technology with robotic operation, it can greatly reduce the cost and time spent on training surgeons due to the requirements of high-level operation skills. The force oscillation may cause deformation of soft tissue and displacement of insertion site, especially when the insertion speed is low, which were observed in manual experiments. To avoid this situation, a relative high speed was defined in robot-assisted system. Due to the liver functions to filter and process blood, it contains a substantial number of arteries and veins, which could explain the peaks following peak 1 and 2 shown in Figure 5. The main puncture event is designated by a peak in force after a steady rise, followed by a sharp decrease. Subsequent variations in force are due to friction, cutting forces, and internal stiffness, as well as collisions with and puncture of interior structures [14].

The bending of bevel tip needle was investigated in our experiments. Allison M. Okamura [14] verified the bending by the X-Ray images and found that the bevel tip needles lead to more needle bending. From their results, increasing needle diameter generally increases the insertion forces but reduces needle bending. From the observation in our experiments, the bending of bevel tip needle shows strict accordance with the state of the arts. By discussing the deformation of liver tissue, it was found that conic tip needle created $45 \%$ larger deformation than bevel tip needle. Although the shape of needle may cause difference of soft tissue deformation [15], in this paper, it was not considered compared with the influence from the needle diameter. Quantitative evaluations of $5 \mathrm{MRI}$ and $5 \mathrm{CT}$ image pairs show that the liver moves rigidly $7.2( \pm 4.2) \mathrm{mm}$ on average, while the remaining non-rigid deformations range from 1.4-3 mm [16]. Compared with the deformation presented in the literatures, estimated deformation given in Figure 6 is reasonable and acceptable. The difference of liver deformation caused by manual operation and robot-assisted operation is under $2 \mathrm{~mm}$ in our tests. Therefore, the manual operation mainly affects the insertion process on insertion force but not on liver deformation.

\section{Conclusion}

A robot-assisted system was developed to improve placement accuracy of needle insertion. Experiments of needle insertion into liver tissue were performed with two kinds of needles, conic tip needle (diameter $8 \mathrm{~mm}$ ) and bevel tip needle (diameter $1.5 \mathrm{~mm}$ ). Manual operation was designed to compare the performance of the presented system. The recorded insertion force varies with the needle diameter, and the bigger diameter causes the increase of insertion force. Either the maximum or average deformation of liver tissue did not show much difference under manual or robot-assisted operation. To conclude, the presented robot-assisted system shows outstanding advantages in improving the controllability and stability during needle insertion into liver by comparing manual operation. In liver surgeries, the skills and experience are critical for surgeons to choose the correct site and control insertion force. The robot-assisted system can greatly reduce the cost and time spent on training surgeons. This study provides comparative demonstration of the advantages to develop robot-assisted needle insertion system.

\section{Acknowledgement}

This work was supported by Fundamental Research Funds for the Central Universities 
(3102015JCS05010), Innovation Foundation for Doctor Dissertation of Northwestern Plolytechnical University (CX201515) and NPU Foundation for Fundamental Research (NPU-FFR-JCY20130118).

\section{References}

[1] J.E.F. Green, S.L. Waters, K.M. Shakesheff, et al., A mathematical model of liver cell aggregation in vitro, Bulletin of Mathematical Biology 71 (2009), 906-930.

[2] E. Roan and K. Vemaganti, Strain rate-dependent viscohyperelastic constitutive modeling of bovine liver tissue, Medical \& Biological Engineering \& Computing 49 (2011), 497-506.

[3] H. Ho, K. Sorrell, A. Bartlett, et al., Modeling the hepatic arterial buffer response in the liver, Medical Engineering \& Physics 35 (2013), 1053-1058.

[4] Y.C. Lu, A.R. Kemper and C.D. Untaroiu, Effect of storage on tensile material properties of bovine liver, Journal of the Mechanical Behavior of Biomedical Materials 29 (2014), 339-349.

[5] C.D. Untaroiu and Y.C. Lu, Material characterization of liver parenchyma using specimen-specific finite element models, Journal of the Mechanical Behavior of Biomedical Materials 26 (2013), 11-22.

[6] H. Rhim, S.N. Goldbert, G.D. Dodd, et al., Essential techniques for successful radio-frequency thermal ablation of malignant hepatic tumors, Radiographics 21 (2001), S17-S35.

[7] R.S. Sedeh, M.T. Ahmadian and F. Janabi-Sharifi, Modeling, simulation, and optimal initiation planning for needle insertion into the liver, Journal of Biomechanical Engineering-Transactions of the Asme 132 (2010), 41001.

[8] C. Gokgol, C. Basdogan and D. Canadinc, Estimation of fracture toughness of liver tissue: Experiments and validation, Medical Engineering \& Physics 34 (2012), 882-891.

[9] E.C. Clarke, S. Cheng, M. Green, et al., Using static preload with magnetic resonance elastography to estimate large strain viscoelastic properties of bovine liver, Journal of Biomechanics 44 (2011), 2461-2465.

[10] E. Mazza, A. Nava, D. Halmloser, et al., The mechanical response of human liver and its relation to histology: An in vivo study, Medical Image Analysis 11 (2007), 663-672.

[11] M. Hurtuk, R.L. Reed, T.J. Esposito, et al., Trauma surgeons practice what they preach: The ntdb story on solid organ injury management, Journal of Trauma-Injury Infection and Critical Care 61 (2006), 243-254.

[12] L. Maier-Hein, S.A. Muller, F. Pianka, et al., Respiratory motion compensation for ct-guided interventions in the liver, Computer Aided Surgery 13 (2008), 125-138.

[13] S.E. Song, N. Hata, I. Iordachita, et al., A workspace-orientated needle-guiding robot for $3 \mathrm{t}$ mri-guided transperineal prostate intervention: Evaluation of in-bore workspace and mri compatibility, International Journal of Medical Robotics and Computer Assisted Surgery 9 (2013), 67-74.

[14] A.M. Okamura, C. Simone and M.D. O'leary, Force modeling for needle insertion into soft tissue, IEEE Transactions on Biomedical Engineering 51 (2004), 1707-1716.

[15] N. Abolhassani, R. Patel and M. Moallem, Needle insertion into soft tissue: A survey, Medical Engineering \& Physics 29 (2007), 413-431.

[16] T. Lange, T.H. Wenckebach, H. Lamecker, et al., Registration of different phases of contrast-enhanced ct/mri data for computer-assisted liver surgery planning: Evaluation of state-of-the-art methods, International Journal of Medical Robotics and Computer Assisted Surgery 1 (2005), 6-20. 